\title{
AVALIAÇÃO DE EQUIPAMENTOS LASER FISIOTERAPÊUTICOS DE EMISSÃO CONTÍNUA E BAIXA POTÊNCIA
}

\author{
Manuela Cristine GELAIN ${ }^{1}$ \\ Elgison da Luz dos SANTOS ${ }^{2}$ \\ Vanessa ERTHAL ${ }^{3}$ \\ Frieda Saicla BARROS ${ }^{4}$ \\ Vicente MACHADO NETO ${ }^{5}$ \\ Percy NOHAMA ${ }^{6 *}$.
}

\footnotetext{
1 : Fisioterapeuta e mestranda do Programa de Pós-Graduação em Engenharia Elétrica e Informática Industrial da Universidade Tecnológica Federal do Paraná (CPGEI - UTFPR). - E-mail: gelainfisio@ gmail.com

2 : Fisioterapeuta e mestrando do Programa de Pós-Graduação em Engenharia Elétrica e Informática Industrial da Universidade Tecnológica Federal do Paraná (CPGEI - UTFPR). - E-mail: elgisantos20@ gmail.com

3 : Fisioterapeuta e doutoranda do Programa de Pós-Graduação em Engenharia Elétrica e Informática Industrial da Universidade Tecnológica Federal do Paraná (CPGEI - UTFPR). - E-mail: acupuntura_vane@ yahoo.com.br

${ }^{4}$ : Doutora em Engenharia - Materiais, Professora do Programa de Pós-Graduação em Engenharia Biomédica (PPGEB UTFPR), saicla@utfpr.edu.br

5 : Doutor em Engenharia Mecânica, Professor do PPGEB - UTFP, - E-mail: vmachado@utfpr.edu.br

${ }^{6}$ : Doutor em Engenharia Elétrica, Professor do CPGEI e PPGEB -UTFPR e Professor titular da Pontifícia Universidade Católica do Paraná (PUCPR), Programa de Pós Graduação em Tecnologia em Saúde (PPGTS). - E-mail: percy.nohama@gmail.com
}

Trabalho desenvolvido no Laboratório de Engenharia de Reabilitação (LER) da Universidade Tecnológica Federal do Paraná (UTFPR), Curitiba, Paraná, Brasil.

*Autor Correspondente: End: Av. Sete de Setembro, 3165, CPGEI/UTFPR - Curitiba/PR - CEP 80230-901. E-mail: acupuntura_vane@yahoo.com.br

\section{Recebido em: 28/05/2014 - Aprovado em: 30/06/2014 - Disponibilizado em: 30/07/2014}

\section{RESUMO}

Laser é uma radiação eletromagnética não ionizante, monocromática, coerente e colimada, sendo utilizado em diferentes aplicações, incluindo a terapia a Laser de baixa potência (TLBP) para o tratamento de doenças. Objetivo: avaliar o desempenho de equipamentos Laser de clínicas de Curitiba-PR, verificando a conformidade dos emissores em relação aos requisitos e potência emitida, descritos nas normas técnicas. Materiais e Métodos: foram selecionados nove emissores, sete equipamentos de três fabricantes distintos. O ensaio seguiu o roteiro de avaliação elaborado por Bertolini e Nohama (2007) referente à coleta de três medidas de potência da radiação emitida por meio de um medidor e o cálculo da média das medidas. Para chegar aos resultados da pesquisa, utilizou-se o programa Minitab16 e o teste ANOVA. Resultados: Dos sete equipamentos avaliados, $71,43 \%$ não apresentaram a etiqueta com saída máxima, duração do pulso e comprimento de onda, que está relacionado à inspeção do equipamento. Na avaliação dos manuais, três itens não foram atendidos. Além dos 
quesitos sobre os protetores de ambiente, nenhum deles cumpriu as exigências e conformidades propostas pela norma. Também foram identificadas alterações nas potências emitidas na maioria dos equipamentos. Conclusão: Os equipamentos de TLBP das marcas verificadas não apresentaram todos os requisitos sugeridos pelas normas, assim como, a emissão de suas potências, o que pode ser prejudicial à saúde de seus usuários.

Palavras-chave: Laser. Laserterapia de baixa potência. Normas técnicas.

\title{
EVALUATION OF LOW-POWER AND CONTINUOUS EMISSION MODE LASER EQUIPMENT
}

\begin{abstract}
Laser is an electromagnetic non-ionizing, monochromatic, coherent and collimated radiation. It has been used on different applications, including the low-level Laser therapy (LLLT) for the treatment of diseases. Objective: to evaluate the performance of Laser devices used at Curitiba-PR clinics, involving the emitters conformity related to technical requirements and output power for safe and effective therapeutics. Methods: there were selected nine emitters, seven Laser devices of three different manufactures. The evaluation essay followed the assessment procedure created by Bertolini e Nohama (2007) and a Laser radiation meter was used to measure three times the equipment output power and to compute its mean value. To calculate the means and to perform ANOVA test we applied the Minitabl6 software. Results: Seven equipments were assessed, $71,43 \%$ did not have the maximum output etiquette, pulse duration, wavelength which they are related with equipment inspection. In the manual's assessment, three parameters were not approved. For the environment protection requirements, none of them fulfilled the requirements and conformities proposed by the standards. There were also identified alterations on the emitted power on almost all equipment. Conclusion: LLLT brand's equipment's verified didn't have all requirements proposal by standards, as well as, the potencies' emission, which could be harmful to health of users.
\end{abstract}

Key words: Laser. Laser therapy low-power. Technical standards.

\section{INTRODUÇÃO}

\section{Laser (Light Amplification by}

Stimulated Emission of Radiation) é uma radiação eletromagnética não ionizante que tem como propriedades a monocromaticidade, a coerência e a colimação (JÚNIOR, OLIVEIRA, FARIAS et al., 2006). Quando de Baixa Potência (LBP), contempla potências menores que $500 \mathrm{~mW}$ (STEFANELLO e HAMERSKI, 2006). O LBP vem sendo utilizado há mais de 30 anos (FUKUDA, JESUS, SANTOS et al., 2010). Desde então, aumentaram as aplicações do LBP em pesquisas experimentais e tratamentos (SILVEIRA, SILVA, TUON et al., 2009) para melhorar sintomas de doenças como exoftalmia, hipertireoidismo, inflamação pélvica e para o alívio da dor (BAXTER, 1994). Os Lasers terapêuticos englobam comprimentos de onda na faixa visível, produzidos a partir de $\mathrm{HeNe}(632,8 \mathrm{~nm})$ e na faixa do infravermelho (730 a 1300nm), fabricados com Arseneto de Gálio (As-Ga) ou Arseneto de Gálio e Alumínio (AsGaAl) (BAXTER, 1994). São comumente encontrados nas clínicas e consultórios (BERTOLINI, CALDEIRA e NOHAMA, 2002; BERTOLINI, 
2003; BERTOLINI e NOHAMA, 2007) como parte do arsenal fisioterapêutico. Sua utilização é justificada por gerar efeito anti-inflamatório e analgésico (GUERINO, BARANAUSKAS, GUERINO et al., 2000); acelerar a proliferação tecidual; aumentar a vascularização local; auxiliar a formação de tecido de granulação organizado; favorecer a cicatrização de úlceras por pressão (STEFANELLO e HAMERSKI, 2006; LOPES, 2011), entre outros. Silveira et al. (2009) demonstraram que a TLBP pode atuar na cadeia respiratória mitocondrial diminuindo a atividade do complexo II, e a produção de ânion superóxido, protegendo a célula contra danos oxidativos em lipídios de membrana. Embora sejam evidentes seus benefícios, ainda existem pontos que precisam ser esclarecidos e padronizados para utilização segura e eficaz. Entre eles, citam-se o comprimento de onda e dose empregada, que podem alterar os efeitos desejados, e os cuidados a serem tomados durante a terapia. Quando o equipamento encontra-se descalibrado ou a aplicação é feita inadequadamente, pode produzir danos à pele ou à visão do paciente e do operador (FUKUDA, JESUS, SANTOS et al., 2010).

Com o intuito de diminuir os riscos e aprimorar o tratamento, está crescendo a atenção voltada ao uso e verificação dos equipamentos médicos e fisioterapêuticos em conformidade com as normas técnicas (PEDROSO e FERREIRA FILHO, 2000), particularmente o Laser, que deve estar em conformidade com as normas NBR IEC 606012-2 (ABNT, 1997) e IEC 60825-1(IEC, 2001) e o relatório TR IEC 60825-8(IEC, 1999). Como fator negativo, no Brasil ainda faltam procedimentos padronizados de ensaios de verificação, laboratórios e pessoas treinadas para realizar os procedimentos (BERTOLINI, CALDEIRA e NOHAMA, 2002; BERTOLINI, 2003; BERTOLINI e NOHAMA, 2007), assim como não há uma grande preocupação por parte dos terapeutas e fabricantes sobre a frequência com que esses equipamentos devem ser submetidos aos serviços de calibração e aferição (FUKUDA, JESUS, SANTOS et al., 2010).

Neste cenário, o objetivo deste estudo foi avaliar uma amostra de equipamentos LBP em uso nas clínicas da cidade de Curitiba, por meio da verificação de conformidade com as prescrições exigidas nas normas técnicas vigentes.

\section{MATERIAIS E MÉTODOS}

O ensaio foi realizado em clínicas e consultórios de fisioterapia na cidade de Curitiba, Paraná. Os estabelecimentos foram localizados nos sítios de busca da internet e 
conhecimentos pessoais dos avaliadores, contatados por telefone ou visita pessoal. $\mathrm{Na}$ seleção dos equipamentos, adotaram-se como critérios de inclusão os emissores Lasers de modo contínuo, em uso na prática fisioterapêutica, com tempo mínimo de utilização de três meses. A avaliação envolveu sete equipamentos com nove emissores, produzidos por três fabricantes distintos. A avaliação seguiu o protocolo de avaliação (ABNT, 1997; IEC, 1999; 2001) desenvolvido por Bertolini e Nohama (2007) incluindo inspeções realizadas no manual do proprietário (item 1 ao 15), no equipamento (item 16 ao 22) e em protetores e ambiente de aplicação (item 23 ao 26):

[1] Informações claras para montagem apropriada, manutenção e utilização segura do equipamento em português;

[2] Advertências claras e concisas sobre precauções para evitar possível exposição à radiação Laser;

[3] Informações claras e de fácil localização no manual sobre a distância nominal de risco ocular;

[4] Declaração da divergência do feixe;

[5] Declaração da duração de pulso;

[6] Declaração da máxima saída Laser;

[7] Reproduções legíveis de todas as etiquetas e avisos de risco afixados ao equipamento;
[8] Informações e orientações para calibração regular;

[9] Especificação do equipamento de medição e a frequência em que o equipamento deve ser calibrado e esclarecimentos sobre as prescrições acerca da calibração regular;

[10] Indicação clara e com tamanho adequado de todas as localizações de abertura do equipamento;

[11] Listagem dos controles, ajustes e procedimentos para operação e manutenção pelo usuário;

[12] Descrição dos sistemas de fornecimento de feixes, incluindo as características da saída;

[13] Nota alertando que o equipamento fora de uso deve ser protegido contra utilização não qualificada;

[14] Especificação clara acerca da proteção dos olhos do operador e paciente;

[15] Informação sobre os riscos potenciais quando inserir, dobrar excessivamente ou segurar de forma inapropriada o cabo de fibra óptica;

[16] Etiqueta posicionada tão próxima quanto possível de cada abertura, similar ao símbolo de perigo do Laser;

[17] Etiqueta explicativa de produto classe 3B e rótulo explicativo dos perigos da exposição a esta classe de Laser;

[18] Etiqueta mostrando saída máxima, duração do pulso e comprimento de onda; 
[19] Indicador de ocorrência de emissão Laser;

[20] Observação do estado dos cabos, principalmente próximos ao gabinete e saída de radiação;

[21] Chave controladora de saída Laser;

[22] Dispositivo de alcance e visualização para desligar o equipamento em casos de urgência; [23] Presença de protetor ocular;

[24] Área controlada de uso do equipamento Laser;

[25] Sinais de aviso sobre advertências na aplicação do Laser na área de uso e

[26] Proteção de janelas.

Para o ensaio da medida de potência do feixe irradiado, utilizou-se o instrumento PocketPower - Handheld Power Meter, produzido pela Melles Griot ${ }^{\circledR}$, com certificado de calibração válido durante os procedimentos. As medidas foram feitas irradiando-se o feixe sobre o sensor do instrumento, que é constituído de uma célula de silício com $8 \mathrm{~mm}$ de diâmetro e exatidão de $\pm 5 \%$. Inicialmente, realizou-se o ajuste do instrumento para o comprimento de onda especificado no equipamento a ser testado. Após o ajuste, o seletor de funções foi posicionado para medida de potência $(\mathrm{mW})$ e, com o equipamento Laser ligado após $15 \mathrm{~s}$ de emissão, irradiou-se sobre o sensor do medidor de potência, por um período mínimo de $2 \mathrm{~s}$. Esse procedimento foi repetido por três vezes, com intervalo de 15 s entre eles.
O valor final considerado foi a média das três medidas de cada emissor, utilizando o programa Minitabl6 para os cálculos e obtenção do $\mathrm{p}$ valor. Todas as medições foram realizadas nos próprios locais de utilização diária dos equipamentos, ocorrendo o escurecimento da sala para evitar interferências luminosas.

\section{RESULTADOS}

A marca A apresentou 1 emissor com comprimento de onda de $660 \mathrm{~nm}$ e 2 emissores com $670 \mathrm{~nm}$. A marca B possui 2 emissores com $660 \mathrm{~nm}$ e 3 com $830 \mathrm{~nm}$. A marca C contem 1 emissor a $830 \mathrm{~nm}$. Desta forma, foram avaliados 7 equipamentos e 9 emissores.

No gráfico 1, a análise da verificação dos manuais indica que apenas 3 equipamentos estavam acompanhados do manual no local de atendimento. Os itens 4, 10 e 13 não foram atendidos por nenhum dos manuais.

Gráfico 1 - Resultados da inspeção do manual dos equipamentos avaliados atendendo aos requisitos.

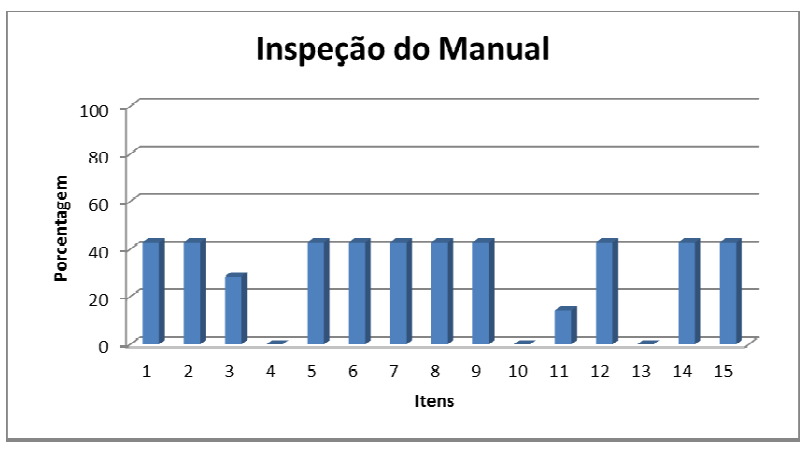

Fonte - Dos próprios autores. 
Os resultados do gráfico 2 referem-se aos itens inspecionados no equipamento, como os cabos próximos ao gabinete e à saída do aparelho e à chave de controle (quesitos 20 e 21). Todos estão de acordo com as recomendações. Três equipamentos $(28,57 \%)$ apresentaram as etiquetas de saída máxima, duração de pulso e comprimento de onda. Ao verificar o ambiente e os equipamentos de proteção, observou-se que a área de aplicação não fornece segurança aos usuários e funcionários.

Gráfico 2 - Resultados da inspeção dos equipamentos, dispositivos de proteção e ambiente de uso, atendendo aos requisitos.

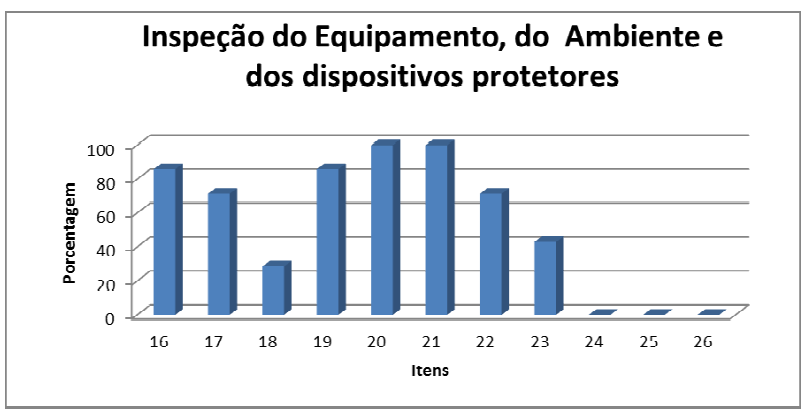

Fonte - Dos próprios autores.

Menos da metade dos locais visitados estava de acordo com o requisito sobre proteção ocular para diferentes comprimentos de onda (item 23). Os itens 24, 25 e 26 não foram encontrados em nenhum local visitado.

A tabela 1 apresenta o número de emissores utilizados nesta pesquisa, suas marcas, suas potências nominas, a média das potências (conforme descrito na metodologia), incerteza do medidor de potência no valor de \pm $5 \%$ e a incerteza menor que $\pm 20 \%$, segundo as normas da Associação Brasileira de Normas Técnicas (ABNT).

A análise de variância ANOVA entre os equipamentos com potência nominal de 30 $\mathrm{mW}$, realizada com o software Minitab 16, para o nível de significância de 95\%, apresentou um $\mathrm{p}$ valor não significativo, indicando que existem diferenças significativas entre as potências dos equipamentos avaliados. Entretanto, ao utilizar a faixa de incerteza $\pm 5 \%$, o total de equipamentos em concordância, segundo o medidor de potência, quando comparado à potência nominal de cada, valida apenas um emissor (C1) descrito na tabela 1 .

Ao utilizar as normas (ABNT, 1997; IEC, 1999; 2001) com incerteza menor que $\pm 20 \%$ (FUKUDA, JESUS, SANTOS et al., 2010), três equipamentos (A1, B1 e B5) com potência nominal de $30 \mathrm{~mW}$ são válidos para utilização e os cinco restantes (A2, A3, B2, B3 e B4) dispõem de valores abaixo da referência. 
Tabela 1 - Comparação da Potência Nominal, Médias das Potências e Faixas de utilização.

\begin{tabular}{|c|c|c|c|c|c|c|}
\hline 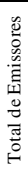 & 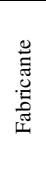 & 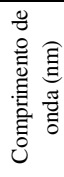 & 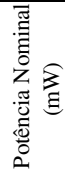 & 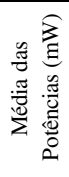 & 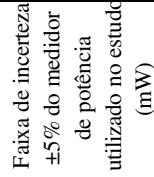 & 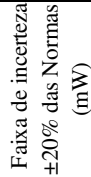 \\
\hline 1 & A1 & 670 & 30 & 27,8 & $28,5-31,5$ & $24-36$ \\
\hline 1 & $\mathrm{~A} 2$ & 670 & 30 & 15,66 & $28,5-31,5$ & $24-36$ \\
\hline 1 & A3 & 660 & 35 & 22,3 & $33,3-36,8$ & $28-42$ \\
\hline 1 & B1 & 660 & 30 & 33,1 & $28,5-31,5$ & $24-36$ \\
\hline 1 & B2 & 660 & 30 & 22,4 & $28,5-31,5$ & $24-36$ \\
\hline 1 & B3 & 830 & 30 & 22,4 & $28,5-31,5$ & $24-36$ \\
\hline 1 & B4 & 830 & 30 & 22,2 & $28,5-31,5$ & $24-36$ \\
\hline 1 & B5 & 830 & 30 & 25,5 & $28,5-31,5$ & $24-36$ \\
\hline 1 & $\mathrm{C} 1$ & 830 & 40 & 40,3 & $38,0-42,0$ & $35-45$ \\
\hline
\end{tabular}

\section{DISCUSSÃO}

Há uma vasta quantidade de equipamentos Lasers desenvolvidos para aplicações médicas, odontológicas, fisioterapêuticas e estéticas. Tais equipamentos deveriam estar em conformidade com as normas técnicas (PANSINI, 2001). No Brasil, a Agência Nacional de Vigilância Sanitária (ANVISA) é o órgão competente para autorizar a fabricação de equipamentos e materiais de saúde, coordenar, acompanhar e avaliar a execução de regulamentos técnicos aplicáveis, planejar e coordenar a execução de programas de controle de qualidade e certificação de produtos (BRASIL, 2001). Além das regulamentações da ANVISA, há normatizações específicas descritas pela ABNT (NBR-IEC 601-2-22) e por órgãos internacionais (IEC TR 60825-1; IEC TR 608258) que devem ser seguidas tanto para a fabricação ou importação dos equipamentos Lasers (EDUARDO E MIRANDA, 1998).
Existem limitações nas reproduções fiéis de estudos utilizando a TLBP devido à falta de desenvolvimento de uma metodologia científica na área e um conflito no relato de parâmetros, tais como: comprimento de onda, irradiação, tempo, propriedades e espessura de pele. A ampla variedade de equipamentos, a qualidade e quantidade de energia emitida pelos mesmos, podem modificar os efeitos desejados (BERTOLINI, 2003). Além disso, o uso prolongado dos equipamentos e o período em que são utilizados nas clínicas de fisioterapia podem gerar deterioração dos dispositivos geradores de radiação, diminuindo a sua eficiência(FUKUDA, JESUS, SANTOS et al., 2010). A manutenção preventiva, a aferição, calibração e verificações periódicas dos equipamentos, bem como o cumprimento dos prazos definidos nos manuais são necessárias. Deste modo, o fisioterapeuta conta com um recurso confiável e seguro para si e para o paciente (GUIRRO e SANTOS, 1997). Caso contrário, a terapia pode não apresentar os efeitos esperados e o profissional pode sofrer sanções éticas (GUIRRO e BRITSHCY DOS SANTOS, 2002; COFFITO, 2013) e legais (EDUARDO e MIRANDA, 1998), pois o paciente pode contratar um serviço/tratamento e não receber o que contratou devido ao erro do equipamento. Neste caso, o paciente é amparado pelo Código de Defesa do 
Consumidor (BRASIL, 2007). Em relação aos manuais, a minoria dos equipamentos estava acompanhada deles, sendo que quatro não os possuíam. Quando os manuais eram encontrados, nem sempre estavam acessíveis. Em um dos locais visitados, o manual encontrava-se em $C D-R O M$, sem qualquer marcação por parte dos profissionais da clínica, o que dificulta a utilização, visto que muitos outros equipamentos também possuíam manual em CD-ROM. Quando os manuais estavam disponíveis, não contemplavam todas as informações obrigatórias e/ou as informações contidas não eram inteligíveis. Isto induz dúvidas no usuário e desestimula a leitura. Este estudo corrobora com o trabalho de Bertolini e Nohama (2007), pois segundo os autores, quando os manuais estavam presentes, havia falta de inúmeros requisitos e informações básicas sobre os equipamentos. Assim, ainda percebe-se certo descaso dos fabricantes quanto ao fornecimento de informação ao profissional que utiliza o equipamento.

$\mathrm{Na}$ inspeção dos equipamentos, a maioria atendeu aos requisitos normativos (NBR-IEC 601-2-22; IEC TR 60825-1; IEC TR 60825-8). Somente o item 18, que buscava a etiqueta com saída máxima, duração do pulso e comprimento de onda não foi encontrado na maioria deles, representando 71,42\%. Há possibilidade de alguns equipamentos possuírem as etiquetas quando novos e, devido ao uso diário podem ter descolado. Por outro lado, é possível que as etiquetas de fato nunca tenham existido no local, denotando o descumprimento das normas por parte dos fabricantes.

O profissional de saúde não tem conhecimento para realizar ajustes técnicos e manutenções de equipamentos terapêuticos (BRANDT e GEWERH, 2002). Assim, faz-se necessário entender que os equipamentos podem sofrer panes e falhar. Por isso, requerem inspeção diária antes de aplicados terapeuticamente, para certificar-se que não há algo diferente no equipamento. Além disso, as manutenções preventivas devem ser incorporadas na sua rotina. Caso o equipamento utilizado não esteja confiável, tudo que resultar de sua aplicação também não o será (COFFITO, 2001). Sugere-se que o profissional crie um checklist diário, anotando as possíveis alterações, fazendo observações minuciosas. Assim, o profissional cria o hábito de checar se todas as etiquetas estão presentes e sem alterações, bem como os cabos, luzes, painel, etc.

Alguns fisioterapeutas não têm o cuidado necessário com a radiação espalhada pelo equipamento no ambiente de aplicação (LOPES, 2011). Por isso, não reforçam a proteção da área de aplicação, evitando e 
controlando $\mathrm{o}$ acesso dos outros usuários $\mathrm{e}$ funcionários. Faz-se necessário colocar no local e nas proximidades, advertências visíveis que há radiação Laser naquele local. $\mathrm{O}$ ambiente não deve ser frequentado por outras pessoas, devido aos riscos à saúde ocular e da pele (BERTOLINI e NOHAMA, 2007; FUKUDA, JESUS, SANTOS et al., 2010). Além disso, não se preocupam em usar os óculos para proteção pessoal e para os pacientes. Também não foi encontrada proteção nas janelas dos locais de aplicação da TLBP, embora na maioria das clínicas o equipamento seja utilizado em gabinetes sem visibilidade, para a privacidade do paciente.

Foram encontrados dados preocupantes com relação à medida de potência da radiação emitida, que estavam acima do valor (B1) ou abaixo (A2). As normas técnicas NBR-IEC 601-2-22; IEC TR 60825-1 e IEC TR 60825-8 determinam que os equipamentos Lasers não apresentem incerteza maior de $\pm 20 \%$ em relação ao valor nominal da potência do fabricante (FUKUDA, JESUS, SANTOS et al., 2010). A maioria dos emissores avaliados neste estudo não atendeu às exigências, podendo ser prejudicial ao paciente. Dos 9 emissores avaliados, apenas 3 estavam de acordo com os padrões normativos, corroborando com os dados de Bertolini e Nohama (2007), que apontavam $61,29 \%$ dos equipamentos avaliados fora dos limites de potência estabelecidos.

Equipamentos em condições funcionais ruins conduzem a terapias com erros dosimétricos, tanto com possibilidade da potência utilizada maior do que a devida ou abaixo daquelas necessárias para a terapia ${ }^{7}$. Por isso, tornam-se necessárias calibrações para evitar que os profissionais utilizem equipamentos sem a dose pretendida. É importante a conscientização dos fisioterapeutas para efetuarem periodicamente as manutenções preventivas dos equipamentos de TLBP, algumas vezes fornecidas pelos fabricantes. Desta maneira, amplia-se a vida útil do equipamento e elevam-se a segurança e o desempenho terapêutico. Não foram avaliados os equipamentos LBP de emissão pulsada, visto que o instrumento empregado no ensaio de potência é específico para emissão contínua.

\section{CONCLUSÃO}

Nenhum equipamento de TLBP das marcas verificadas apresentou todos os requisitos em conformidade com as normas. A maioria também não apresentou conformidade em relação à potência emitida, o que pode ser prejudicial à saúde, em especial, a ocular, do paciente e do profissional que o utiliza. A partir dos resultados obtidos, constatou-se a 
necessidade de realização de processos de calibração e inspeção dos equipamentos periódica e constantemente, para que a TLBP contemple os objetivos preconizados. Os fisioterapeutas que não estão habituados a checar os manuais dos equipamentos e que não fazem a calibração periódica da emissão do Laser, podem estar utilizando os equipamentos fora dos padrões recomendados. É urgente que se fiscalize os fornecedores no cumprimento das normas, o aperfeiçoamento dos manuais, sendo mais explicativos e de fácil entendimento, para melhorar a qualidade e a segurança dos atendimentos, e a saúde e bem estar dos usuários. Sugere-se um estudo multicêntrico, no Brasil todo, a fim de contemplar um universo maior de equipamentos incluindo os equipamentos de emissão pulsada, que não foram alvo deste estudo.

\section{REFERÊNCIAS}

ABNT. NBR IEC 601-2-22 Equipamento eletromédico: prescrições particulares para a segurança de equipamento terapêutico e de diagnóstico a Laser. . Associação Brasileira De Normas Técnicas Rio de Janeiro (RJ) 1997.

BAXTER, G. D. Therapeutic Lasers theory and pratice. 1994
BERTOLINI, G. Proposta de ensaios e avaliação de equipamentos Laser de baixa potência empregados em fisioterapia. Programa de Pós-graduação em Engenharia Elétrica e Informática Industrial, Centro Federal de Educação Tecnológica do Paraná, Curitiba, 2003.

BERTOLINI, G., CALDEIRA, L. e NOHAMA, P. Proposta de Avaliação dos Equipamentos de Laserterapia Baseada nas Normas IEC 60601-2-22, IEC 60825-1 e IEC 60825-8. In: (Ed.), Anais do $\mathbf{1 8}^{\mathbf{0}}$ Congresso Brasileiro de Engenharia Biomédica CBEB. São José dos Campos. p.6, 2002.

BERTOLINI, G. e NOHAMA, P. Avaliação da conformidade dos equipamentos laser de baixa potência e emissão contínua empregados em fisioterapia. Fisioterapia em Movimento, v.20, n.2, p.13-23, 2007.

BRANDT, A. e GEWERH, P. Programa de verificação da qualidade de equipamentos de ultra-som terapêuticos. Anais do Metrosul. Curitiba 2002.

BRASIL. AGÊNCIA NACIONAL DE VIGILÂNCIA SANITÁRIA. Manual do Usuário da Resolução-RDC $\mathrm{n}^{\circ} \quad$ 185/2001. 
Orientações sobre registro, cadastramento, alteração, revalidação e cancelamento do registro de produtos: ANVISA, 2001.

BRASIL. LEI $\mathrm{N}^{\circ} \quad 8.078, \quad$ DE $11 \quad \mathrm{DE}$ SETEMBRO DE 1990. Dispõe sobre a proteção do consumidor e dá outras providências. Publicado em DOU 12.9.1990, Retificado no DOU de 10.1.2007 2007.

COFFITO. Equipamentos de fisioterapia: ordem no caos. O COFFITO. CONSELHO FEDERAL DE FISIOTERAPIA E TERAPIA OCUPACIONAL: 23-26 p. 2001.

COFFITO. RESOLUÇÃO Nº24, DE 08 DE JULHO DE 2013, Estabelece o Código de Ética e Deontologia da Fisioterapia, . CONSELHO FEDERAL DE FISIOTERAPIA E TERAPIA OCUPACIONAL. D.O.U. $\mathrm{n}^{\circ} 147$, CONSELHO FEDERAL DE FISIOTERAPIA E TERAPIA OCUPACIONAL 2013.

EDUARDO, M. B. P. e MIRANDA, I. C. S. Saúde \& Cidadania-vigilância sanitária. Instituto para o Desenvolvimento da SaúdeIDS. Núcleo de Assistência MédicoHospitalar-NAMH/FSP e Banco Itaú. São Paulo, p.3, 1998.
FUKUDA, T. Y., JESUS, J. F., SANTOS, M. G., JUNIOR, C. C., TANJI, M. M. e PLAPLER, H. Aferição dos equipamentos de laser de baixa intensidade. Revista Brasileira de Fisioterapia, v.14, n.4, p.303-308, 2010.

GUERINO, M., BARANAUSKAS, V., GUERINO, A. e PARIZOTTO, N. Laser treatment of experimentally induced chronic arthritis. Applied surface science, v.154, p.561-564, 2000.

GUIRRO, R. e BRITSHCY DOS SANTOS, S. C. Evaluation of the acoustic intensity of new ultrasound therapy equipment. Ultrasonics, v.39, n.8, p.553-557, 2002.

GUIRRO, R. e SANTOS, S. A realidade da potência acústica emitida pelos equipamentos de ultra-som terapêutico: uma revisão. Rev Fisioter Univ São Paulo, v.4, n.2, p.76-82, 1997.

IEC. IEC TR 60825-8: Safety of Laser products: guidelines for the safe use of medical Laser equipment. Switzerland, 1999. 
IEC. IEC 60825-1: Safety of Laser products: equipment classification, requirements and user's guide. Switzerland, 2001.

JÚNIOR, A. R., OLIVEIRA, R., FARIAS, R. E., ANDRADE, L. e AARESTRUP, F. M. Modulação da proliferação fibroblástica e da resposta inflamatória pela terapia a laser de baixa intensidade no processo de reparo tecidual. An Bras Dermatol, v.81, n.2, p.1506, 2006.

LOPES, L. D. F. Utilização do laser de 660 nm, $17 \mathrm{~J} / \mathrm{cm} 2$ em úlceras por pressão- Um relato de caso. Revista Neurociências, n.in press, 2011.

PANSINI, M. Avaliação de requisitos de segurança em lasers terapêuticos de baixa intensidade. Instituto de Pesquisas Energéticas e Nucleares, Faculdade de Odontologia da Universidade de São Paulo, Universidade de São Paulo, São Paulo (SP), 2001.
PEDROSO, J. e FERREIRA FILHO, J. Desenvolvimento de um programa de segurança elétrica e avaliação de desempenho para equipamentos eletromédicos baseado nas normas da família NBR IEC 60601. Anais do $17^{\circ}$ Congresso Brasileiro de Engenharia Biomédica - CBEB. Florianópolis 2000.

SILVEIRA, P., SILVA, L., TUON, T., FREITAS, T., STRECK, E. e PINHO, R. Efeitos da laserterapia de baixa potência na resposta oxidativa epidérmica induzida pela cicatrização de feridas. Rev Bras Fisioter, v.13, n.4, p.281-7, 2009.

STEFANELLO, T. D. e HAMERSKI, C. R. Tratamento de úlcera de pressão através do laser AsGa de 904 nm-um relato de caso. Arquivos de Ciências da Saúde da Unipar, Umuarama, v.10, n.2, p.99-103, 2006. 